\title{
Chimera States in a Ring of Nonlocally Coupled Oscillators
}

\author{
Daniel M. Abrams and Steven H. Strogatz \\ Niels Bohr Institute, Blegdamsvej 17, 2100 Copenhagen Ø, Denmark*
}

\begin{abstract}
Arrays of identical limit-cycle oscillators have been used to model a wide variety of patternforming systems, such as neural networks, convecting fluids, laser arrays, and coupled biochemical oscillators. These systems are known to exhibit rich collective behavior, from synchrony and traveling waves to spatiotemporal chaos and incoherence. Recently, Kuramoto and his colleagues reported a strange new mode of organization - here called the chimera state - in which coherence and incoherence exist side by side in the same system of oscillators. Such states have never been seen in systems with either local or global coupling; they are apparently peculiar to the intermediate case of nonlocal coupling. Here we give an exact solution for the chimera state, for a one-dimensional ring of phase oscillators coupled nonlocally by a cosine kernel. The analysis reveals that the chimera is born in a continuous bifurcation from a spatially modulated drift state, and dies in a saddle-node collision with an unstable version of itself.
\end{abstract}

\footnotetext{
* Permanent Address: 212 Kimball Hall, Department of Theoretical and Applied Mechanics, Cornell University, Ithaca, NY 14853-1503, USA
} 


\section{INTRODUCTION}

\section{A. The chimera state}

A fascinating spatiotemporal pattern was reported recently by Kuramoto, Battogtokh and Shima [10, 11, 18]. While studying arrays of identical limit-cycle oscillators that are coupled nonlocally, they found that for certain choices of parameters and initial conditions, the array would split into two domains: one composed of coherent, phase-locked oscillators, coexisting with another composed of incoherent, drifting oscillators. The coexistence of locking and drift was robust. It occurred in both one and two spatial dimensions, and for various kinds of oscillators, including the Fitzhugh-Nagumo model, complex Ginzburg-Landau equations, phase oscillators, and an idealized model of biochemical oscillators.

It's important to appreciate how unexpected this coexistence state was. Nothing like it had ever been seen before, at least not in an array of identical oscillators. Normally, identical oscillators settle into one of a few basic patterns [4, 8, 27]. The simplest is synchrony, with all oscillators moving in unison, executing identical motions at all times. Another common pattern is wave propagation, typically in the form of solitary waves in one dimension, spiral waves in two dimensions, and scroll waves in three dimensions. The common feature in these cases is that all the oscillators are locked in frequency, with a fixed phase difference between them. At the opposite end of the spectrum is incoherence, where the phases of all the oscillators drift quasiperiodically with respect to each other, and the system shows no spatial structure whatsoever. And finally, one sometimes sees more complex patterns, including modulated structures, spatiotemporal chaos, intermittency and so on.

What was so odd about the coexistence state is that two of these patterns (locking and incoherence) were present in the same system, simultaneously. This combination of states couldn't be ascribed to the simplest mechanism of pattern formation - a supercritical instability of the spatially uniform oscillation-because it can occur even if the uniform state is linearly stable, as indeed it was for the parameter values used by Kuramoto and his colleagues. Furthermore, it has nothing to do with the classic partially locked/partially incoherent states that occur in populations of non-identical oscillators with distributed natural frequencies [8, 26]. There, the splitting of the population stems from the inhomogeneity of the oscillators themselves; the desynchronized oscillators are the intrinsically fastest and 
slowest ones in the tails of the distribution. In contrast, for the system studied by Kuramoto et al., there is no distribution of frequencies. All the oscillators are the same, and yet they still break apart into two groups of utterly different character.

Because the coexistence state involves two seemingly incompatible forms of dynamical behavior, we will henceforth refer to it as "the chimera state," inspired by the mythological creature composed of a lion's head, a goat's body, and a serpent's tail. Today the word chimera is used more generally to indicate something made up of incongruous parts, or something that seems wildly improbable or fantastical.

Figure 1 shows a realization of the chimera state in the simplest setting, a one-dimensional ring of phase oscillators $[10,11]$. The governing equation is

$$
\frac{\partial \phi}{\partial t}=\omega-\int_{0}^{1} G\left(x-x^{\prime}\right) \sin \left[\phi(x, t)-\phi\left(x^{\prime}, t\right)+\alpha\right] d x^{\prime}
$$

where $\phi(x, t)$ is the phase of the oscillator at position $x$ at time $t$. The space variable $x$ runs from 0 to 1 with periodic boundary conditions, and should be regarded as an angle on a circle $(\bmod 1)$. The frequency $\omega$ plays no role in the dynamics, in the sense that one can set $\omega=0$ without loss of generality by redefining $\phi \rightarrow \phi+\omega t$, without otherwise changing the form of equation (11). The kernel $G\left(x-x^{\prime}\right)$ provides nonlocal coupling between the oscillators. It is assumed to be even, non-negative, decreasing with the separation $\left|x-x^{\prime}\right|$ along the ring, and normalized to have unit integral. Specifically, Kuramoto and Battogtokh [10, 11] used an exponential kernel $G\left(x-x^{\prime}\right) \propto \exp \left(-\kappa\left|x-x^{\prime}\right|\right)$. Then, for parameter values $\alpha=1.457$ and $\kappa=4$ and suitable initial conditions (to be discussed in detail in Section III), the system evolves to the chimera state shown in Fig. [1.

In this snapshot of the instantaneous phases, two distinct regions are conspicuous. The oscillators near $x=0$ (and equivalently, $x=1$ ) are phase-locked. All of them move with the same constant frequency; in a frame rotating at this frequency, they would all look frozen. The smoothness and flatness of the graph of $\phi(x)$ in this region indicates that these oscillators are coherent as well, i.e., they are nearly in phase.

Meanwhile, the scattered oscillators in the middle of Fig. 1 are drifting, both with respect to each other and with respect to the locked oscillators. Their motion is strongly nonuniform. They slow down when they pass near the locked pack - which is why the dots appear more densely clumped at this phase - and then speed up as they lap it. 


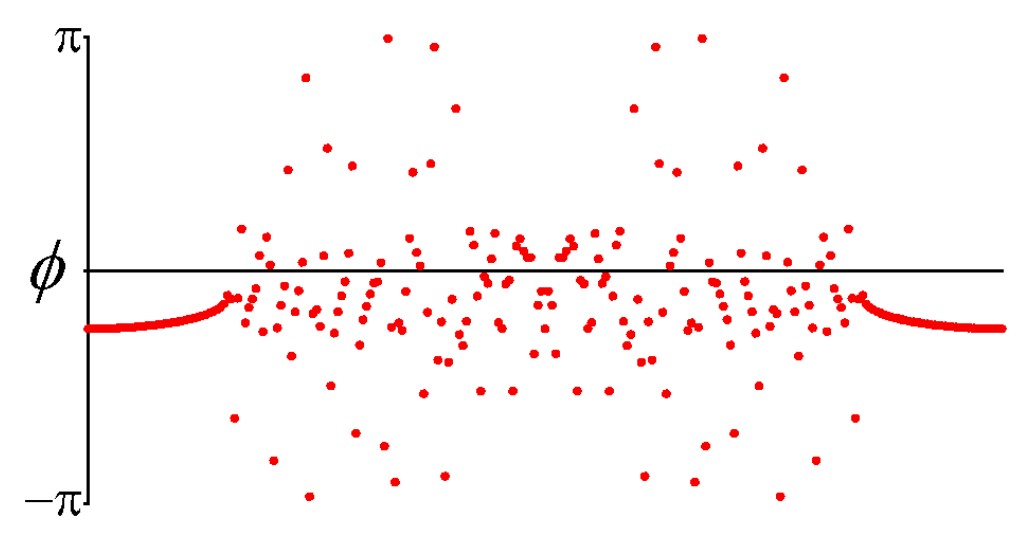

FIG. 1: Phase pattern for a typical chimera state. Here $\kappa=4.0, \alpha=1.45, N=256$ oscillators. Equation (11) was integrated with fixed time step $d t=0.025$ for 8,000 iterations, starting from $\phi(x)=6 \exp \left[-30\left(x-\frac{1}{2}\right)^{2}\right] r(x)$, where $r$ is a uniform random variable on $\left[-\frac{1}{2}, \frac{1}{2}\right]$.

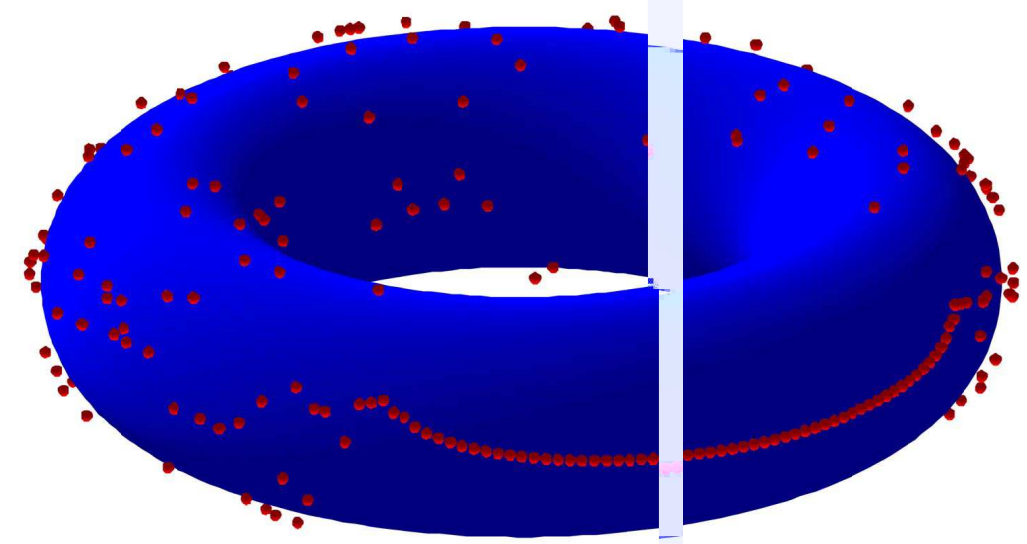

FIG. 2: Phase pattern for a typical chimera state shown on the torus. Azimuthal angle indicates spatial position $x$. Phase $\phi$ is constant along lines of latitude; the outer equator of the torus corresponds to $\phi=0$.

\section{B. Puzzles}

When we first learned about the chimera state by reading Ref. [10], we were amazed by it. How could such a thing even be possible?

In fact, a little thought showed that it was provably impossible in two special cases that had been studied previously:

- Global coupling: Chimera states can't occur for Eq. (11) with $G(x) \equiv 1$ and any choice of $0 \leq \alpha<\pi / 2$, because a Lyapunov function exists for this case, demonstrating that 
almost all solutions are attracted to the in-phase oscillation [23, 24].

- Sine coupling: If $\alpha=0$, corresponding to a pure sine coupling in Eq. (11), chimera states are impossible for any even kernel $G$ of any range. This follows because Eq. (1) becomes a gradient system in the frame rotating at frequency $\omega$. Hence all attractors must be fixed points, corresponding to phase-locked solutions in the original frame, thus ruling out the possibility of coexisting drift.

So the coexistence phenomenon must somehow rely on a conspiracy between $\alpha \neq 0$ and the non-global nature of the coupling. But how, exactly?

And for that matter, is the chimera state born as soon as $\alpha \neq 0$, or at some value of $\alpha$ bounded away from zero? In dynamical simulations like that shown in Fig. 11, stable chimera states are observed only when $\alpha$ is close to, but slightly less than, $\pi / 2$. Does that mean that these states don't exist for smaller $\alpha$, or is just that their basins of attraction shrink as $\alpha$ decreases?

Furthermore, what is the genealogy of the chimera state, in the sense of bifurcation theory? Is it born out of the vacuum, as a pair of stable and unstable versions of itself? Or does it emerge when some more familiar attractor loses stability? For instance, does it bifurcate off the fully incoherent state, in which oscillators are uniformly scattered and drifting around the circle at every $x$ ? That seems unlikely, since the phase pattern shown in Fig. 3 looks pretty far from total incoherence; even its drifting oscillators show some clumping in phase. So maybe the chimera state branches off the uniform in-phase state? But how can it, given that the in-phase state is linearly stable for all $|\alpha|<\frac{\pi}{2}$ ?

Motivated by these puzzles, we have tried to understand where the chimera state comes from and to pinpoint the conditions that allow it to exist. A brief report of our findings appeared in [1].

\section{Broader significance}

Aside from the questions it raises, we believe the chimera state is also more broadly significant for nonlinear science, for two reasons.

First, it exemplifies the surprises that lurk in nonlocally coupled systems. As Kuramoto and his colleagues have pointed out [2, 9, 10, 11, 12, 18, 22], nonlocal coupling is a relatively 
dark corner of nonlinear science in general, and nonlinear oscillator theory in particular. Most previous work on coupled oscillators has focused on local coupling, where the interactions are assumed to be solely between nearest neighbors, or global coupling, where each oscillator interacts equally strongly with all the others. The intermediate case of nonlocal coupling is natural to explore next, and has already revealed some interesting new forms of dynamical behavior [9, 12].

From a more applied perspective, nonlocal coupling is important to investigate because it arises in diverse systems throughout physics, chemistry, and biology. Examples include Josephson junction arrays [17], chemical oscillators [8], epidemiological models of disease spread [15], and the neural networks underlying the patterns on mollusc shells [6, 16], localized neural "bump" states [3, 7, 13], and ocular dominance stripes in the visual cortex [16, 21].

Second, the chimera state is by no means an oddity restricted to Eq. (11). On the contrary, it was first seen in simulations of the complex Ginzburg-Landau equation with nonlocal coupling [10, 11], a fundamental model in the study of pattern formation. That equation in turn can be systematically derived from a wide class of reaction-diffusion equations, under particular assumptions on the local kinetics and diffusion strength that render the effective coupling nonlocal 10, 11, 18, 22]. Under an additional assumption that the coupling is also sufficiently weak (in a precise sense), Shima and Kuramoto 18] show that the original reaction-diffusion system can be further reduced to a phase equation of the universal form

$$
\frac{\partial \phi}{\partial t}=\omega-\int d \boldsymbol{r}^{\prime} G\left(\boldsymbol{r}-\boldsymbol{r}^{\prime}\right) \sin \left[\phi(\boldsymbol{r})-\phi\left(\boldsymbol{r}^{\prime}\right)+\alpha\right]
$$

where $\boldsymbol{r}$ labels the position of the oscillators and the kernel $G$ decays exponentially with distance: $G\left(\boldsymbol{r}-\boldsymbol{r}^{\prime}\right) \propto \exp \left(-\kappa\left|\boldsymbol{r}-\boldsymbol{r}^{\prime}\right|\right)$. But this is just Eq. (11), if the space is onedimensional. So there is good reason to expect that the coexistence phenomenon will have some generality.

For example, in two dimensions, the coexistence of locked and drifting oscillators manifests itself as an unprecedented kind of spiral wave: one without a phase singularity at its center [10, 18]. Instead, the oscillators in the core are found to be completely desynchronized from each other and from the uniform rotation of the spiral arms. In effect, the core oscillators mimic a phase singularity by being incoherent. A better understanding of the one-dimensional case might shed light on this remarkable new form of pattern formation. 


\section{SUMMARY OF PRIOR RESULTS}

We begin by reviewing the results of Kuramoto and Battogtokh [10,11]. After uncovering the chimera state in their simulations of Eq. (II), they were able to explain much of its structure analytically. Their elegant approach is a generalization of Kuramoto's self-consistency argument for globally coupled oscillators [8, 19].

In this approach, one first transforms (11) by seeking a rotating reference frame in which the dynamics become as simple as possible. Let $\Omega$ denote the angular frequency of this rotating frame (to be determined later, in the course of solving the problem), and let

$$
\theta=\phi-\Omega t
$$

denote the phase of an oscillator relative to this frame. Next, introduce a complex order parameter $R e^{i \Theta}$ that depends on space and time:

$$
R(x, t) e^{i \Theta(x, t)}=\int_{0}^{1} G\left(x-x^{\prime}\right) e^{i \theta\left(x^{\prime}, t\right)} d x^{\prime} .
$$

To see what this order parameter means intuitively, note that the integral on the right hand side of (2) performs a running average of $e^{i \theta}$ over a window centered at $x$, with a width determined by the width of the kernel $G$. Thus $0 \leq R(x, r) \leq 1$ can be viewed as a measure

of the local phase coherence at $x$, and $\Theta(x, t)$ represents the local average phase. These two average quantities provide macroscopic proxies for the overall state of the continuum of oscillators.

The real virtue of introducing the order parameter, however, is that we can now rewrite the governing equation (11) as

$$
\frac{\partial \theta}{\partial t}=\omega-\Omega-R \sin [\theta-\Theta+\alpha]
$$

which makes it look as if the oscillators have decoupled, though of course they are still interacting through $R$ and $\Theta$, to which they each contribute through (2). This observation suggests that the problem can be attacked by the self-consistency arguments of mean-field theory, even though it is not globally coupled.

Now comes the key step. Suppose we restrict attention to stationary solutions, in which $R$ and $\Theta$ depend on space but not on time. Now the equations truly do decouple, in the following sense. One can easily solve for the motion of the oscillator at each $x$, subject to 
the assumed time-independent values of $R(x)$ and $\Theta(x)$. The oscillators with $R(x) \geq|\omega-\Omega|$ asymptotically approach a stable fixed point $\theta^{*}$, defined implicitly by

$$
\omega-\Omega=R(x) \sin \left[\theta^{*}-\Theta(x)+\alpha\right]
$$

The fact that they approach a fixed point in the rotating frame implies that they are phase-locked at frequency $\Omega$ in the original frame. On the other hand, the oscillators with $R(x)<|\omega-\Omega|$ drift around the phase circle monotonically. To be consistent with the assumed stationarity of the solution, these oscillators must distribute themselves according to an invariant probability density $\rho(\theta)$. (To ease the notation here and elsewhere, we have suppressed the dependence on $x$ whenever it's clear from context.) And for the density to be invariant, the probability of finding an oscillator near a given value of $\theta$ must be inversely proportional to the velocity there. From (13), this condition becomes

$$
\rho(\theta)=\frac{\sqrt{(\omega-\Omega)^{2}-R^{2}}}{2 \pi|\omega-\Omega-R \sin (\theta-\Theta+\alpha)|}
$$

where the normalization constant has been chosen such that $\int_{-\pi}^{\pi} \rho(\theta) d \theta=1$.

The resulting motions of both the locked and drifting oscillators must be consistent with the assumed time-independent values for $R(x)$ and $\Theta(x)$. To calculate the contribution that the locked oscillators make to the order parameter (2), observe that

$$
\begin{aligned}
\sin \left(\theta^{*}-\Theta+\alpha\right) & =\frac{\omega-\Omega}{R} \\
\cos \left(\theta^{*}-\Theta+\alpha\right) & = \pm \frac{\sqrt{R^{2}-(\omega-\Omega)^{2}}}{R}
\end{aligned}
$$

for any fixed point of (3). One can check that the stable fixed point of (3) corresponds to the plus sign in (6) . Hence

$$
\exp \left[i\left(\theta^{*}-\Theta+\alpha\right)\right]=\frac{\sqrt{R^{2}-(\omega-\Omega)^{2}}+i(\omega-\Omega)}{R}
$$

which implies that the locked oscillators contribute

$$
\int d x^{\prime} G\left(x-x^{\prime}\right) \exp \left[i \theta^{*}\left(x^{\prime}\right)\right]=e^{-i \alpha} \int d x^{\prime} G\left(x-x^{\prime}\right) \exp \left[i \Theta\left(x^{\prime}\right)\right] \frac{\sqrt{R^{2}-(\omega-\Omega)^{2}}+i(\omega-\Omega)}{R}
$$

to the order parameter (2). Here the integral is taken over the portion of the domain where $R\left(x^{\prime}\right) \geq|\omega-\Omega|$. 
Next, to calculate the contribution from the drifting oscillators, Kuramoto and Battogtokh [10, 11] replace $\exp \left[i \theta\left(x^{\prime}\right)\right]$ in (2) with its statistical average $\int_{-\pi}^{\pi} \exp (i \theta) \rho(\theta) d \theta$. Using (15) and contour integration, they obtain

$$
\int_{-\pi}^{\pi} \exp (i \theta) \rho(\theta) d \theta=\frac{i}{R}\left(\omega-\Omega-\sqrt{(\omega-\Omega)^{2}-R^{2}}\right) .
$$

Therefore the contribution of the drifting oscillators to the order parameter is

$$
\int d x^{\prime} G\left(x-x^{\prime}\right) \int_{-\pi}^{\pi} \exp (i \theta) \rho(\theta) d \theta=i e^{-i \alpha} \int d x^{\prime} G\left(x-x^{\prime}\right) \exp \left[i \Theta\left(x^{\prime}\right)\right] \frac{\omega-\Omega-\sqrt{(\omega-\Omega)^{2}-R^{2}\left(x^{\prime}\right)}}{R\left(x^{\prime}\right)}
$$

where now the integral is over the complementary portion of the domain where $R\left(x^{\prime}\right)<$ $|\omega-\Omega|$.

Notice something curious: the integrand on the right hand side of the drifting contribution is exactly the same as that found earlier in (8) for the locked contribution; only their domains differ. (This coincidence is not mentioned in [10,11].) To see that the two expressions agree, note that

$$
\sqrt{R^{2}-(\omega-\Omega)^{2}}+i(\omega-\Omega)=i\left(\omega-\Omega-\sqrt{(\omega-\Omega)^{2}-R^{2}}\right)
$$

as long as we choose the branch corresponding to the " $+i$ " square root of a negative number.

Hence the two contributions can be combined into a single integral, yielding a slightly more compact version of the self-consistency equation derived in [11]:

$$
R(x) \exp [i \Theta(x)]=i e^{-i \alpha} \int_{0}^{1} G\left(x-x^{\prime}\right) \exp \left[i \Theta\left(x^{\prime}\right)\right] \frac{\omega-\Omega-\sqrt{(\omega-\Omega)^{2}-R^{2}\left(x^{\prime}\right)}}{R\left(x^{\prime}\right)} d x^{\prime} .
$$

To ease the notation a bit more, let

$$
\begin{aligned}
\beta & =\frac{\pi}{2}-\alpha \\
\Delta & =\omega-\Omega .
\end{aligned}
$$

Then the self-consistency equation becomes

$$
R(x) e^{i \Theta(x)}=e^{i \beta} \int_{0}^{1} G\left(x-x^{\prime}\right) e^{i \Theta\left(x^{\prime}\right)} \frac{\Delta-\sqrt{\Delta^{2}-R^{2}\left(x^{\prime}\right)}}{R\left(x^{\prime}\right)} d x^{\prime} .
$$

Equation (10) is to be solved for three unknowns - the real-valued functions $R(x)$ and $\Theta(x)$ and the real number $\Delta$-in terms of the assumed choices of $\beta$ and the kernel $G(x)$. Notice that although $\omega$ itself is arbitrary up to a constant, and hence so is $\Omega$, their difference 
$\omega-\Omega$ is physically meaningful; it is determined by the condition that the long-term dynamics become stationary in the frame rotating at frequency $\Omega$.

Initially, we couldn't see how to solve the self-consistency equation (10) numerically. We wrote to Kuramoto for advice, and he described an iterative scheme to determine the functions $R(x)$ and $\Theta(x)$, based on initial guesses obtained from the dynamical simulations. The idea behind the scheme is that the current estimates of $R(x)$ and $\Theta(x)$ can be entered into the right hand side of (10), and used to generate the new estimates appearing on the left hand side.

Still, that leaves open the question of how to determine $\Delta$. We seem to have only two equations (given by the real and imaginary parts of Eq. (10i)) for three unknowns. Fortunately, a third equation can be imposed to close the system. Because (10) is left unchanged by any rigid rotation $\Theta(x) \rightarrow \Theta(x)+\Theta_{0}$, we can specify the value of $\Theta(x)$ at any point $x$ we like; this freedom is tantamount to choosing an origin in the rotating frame. A natural choice would be to demand $\Theta(0)=0$, but as we'll see in Section IV another choice turns out to be more convenient.

Kuramoto and Battogtokh [10, 11] confirm that the self-consistency approach works: their results from numerical integration of the dynamical equations (11) match those obtained by solving the self-consistency equation (10) iteratively.

Figure 3 shows the chimera state along with the graphs of $R(x)$ and $\Theta(x)$ for the parameters used in Fig. 10 The curves in Fig. [3(b) and [3(c) are periodic and reflection-symmetric. In fact, they resemble cosine waves, which made us wonder whether (10) might have a simple closed-form solution, perhaps in some perturbative limit as a parameter tends to zero. To see where such a limit might come into play, we hoped to first replicate the simulations of Kuramoto and Battogtokh [10, 11] and then to explore parameter space more widely.

\section{A FIRST ROUND OF SIMULATIONS}

Unfortunately, we couldn't find the chimera state in our early simulations of Eq. (11). No matter how we started the system, it always converged to the in-phase state. In the report that announced the chimera state, Kuramoto [10] does not give precise details of the initial condition he used. He describes it as a "suitable single-humped initial phase pattern" 10, p. 219] which we incorrectly took to mean something like $\phi(x, 0)=a+b \cos x$ or $e^{-a \cos x}$. 


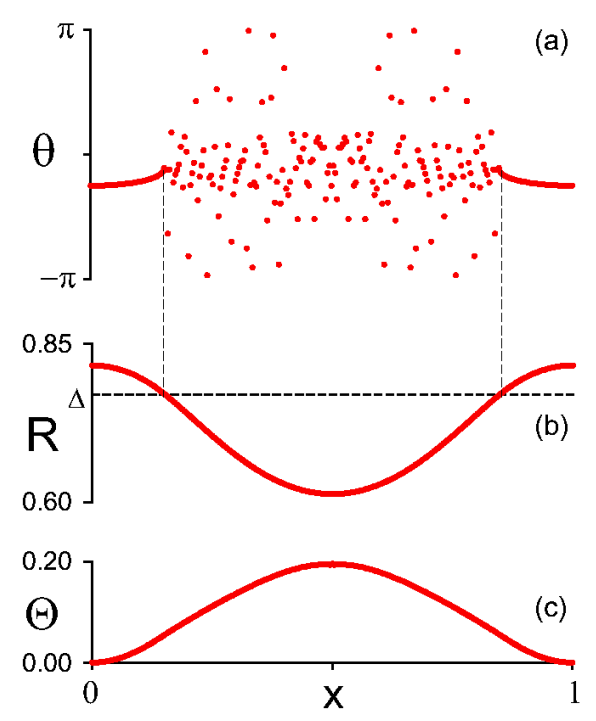

FIG. 3: Chimera state and order parameter curves for the exponential kernel $G(x) \propto \exp (-\kappa|x|)$, as used by Kuramoto and Battogtokh [10, 11]. Parameters are the same as those in Fig. 1] (a) Phase pattern for chimera state. (b) Local phase coherence $R(x)$, computed from (2). Locked oscillators satisfy $R(x) \geq \Delta$. (c) Local average phase $\Theta(x)$, computed from (2).

Eventually, we asked Kuramoto for help (again!), and he kindly explained what he meant. (He also sent us his paper [11] with Battogtokh, where the description of the initial condition is more explicit.) At each $x$, a uniform random number $\phi(x, 0)$ is chosen within some interval whose width varies with $x$ in a single-humped fashion. Specifically, the width is narrowest near $x=0(\bmod 1)$, meaning that the oscillators are most nearly in phase there, initially. As $x$ increases toward the diametrically opposite point of the domain at $x=\frac{1}{2}$, the phases are scattered progressively over larger and larger regions on the phase circle (meaning the oscillators are placed more and more incoherently there, initially). The effect of this procedure is to give the system a jump-start, by placing it in a partially coherent/partially incoherent state to begin with.

To be more precise, Kuramoto used a random distribution with a Gaussian envelope: $\phi(x, 0)=6 \exp \left[-30\left(x-\frac{1}{2}\right)^{2}\right] r(x)$, where $r(x)$ is a uniform random number on the interval $-\frac{1}{2} \leq r \leq \frac{1}{2}$. For the parameters used in Fig. 3, this initial condition indeed evolves to the chimera state reported in [10, 11].

Then we ran simulations to see how far this state could be continued by decreasing $\alpha$, knowing that it would have to disappear or lose stability somewhere before $\alpha=0$. To track 
its fate along the way, we also computed several statistics:

1. the spatial average of $R(x)$, given by $\langle R\rangle=\int_{0}^{1} R(x) d x$;

2. the amplitude of $R(x)$, defined as $R_{a m p}=R_{\max }-R_{\min }$;

3. $f_{\text {drift }}$, the fraction of the spatial domain occupied by drifting oscillators;

4. the difference $\Delta=\omega-\Omega$ between the nominal frequency of individual oscillators and their collective frequency when locked; and

5. $\bar{\Delta}_{\max }$, the largest value of the time-averaged drift velocity relative to the rotating frame. This quantity measures the average speed of the fastest drifting oscillator. From (13), it can be calculated as $\max _{x}|\bar{\Delta}(x)|=\max _{x}\left|\sqrt{\Delta^{2}-R^{2}(x)}\right|$, where the maximum is taken only over the drifting oscillators.

Figure 4 shows how $f_{\text {drift }}$ varies when $\kappa$ is held constant but $\alpha$ is changed smoothly. We generated similar graphs for each of the statistics mentioned above, and all showed a jump to the uniform synchronized state as $\alpha$ decreased below some critical value $\alpha_{c}$. From these results it appeared that when $\kappa=4.0$, the chimera state ceased to exist somewhere around $\alpha_{c} \approx 1.37$. The transition seemed to be discontinuous, which suggested that $\alpha_{c}$ couldn't be calculated by a naive perturbation expansion. If it was to be calculable at all, something more subtle would be required.

The next step was to investigate how these results vary with $\kappa$. Recall that the kernel in (10) is $G(x)=C \exp (-\kappa|x|)$, so $1 / \kappa$ sets a characteristic length scale. Roughly speaking, it is the distance over which the nonlocal coupling is substantial. So the limit $\kappa \rightarrow 0^{+}$ corresponds to global coupling $G(x) \equiv 1$. This can also be checked directly, noting that the normalization constant for the exponential kernel on the circle is given by $C=\frac{\kappa}{2}\left(1-e^{-\kappa / 2}\right)^{-1}$.

Figure 5 shows a rough contour plot of $R_{a m p}$ in the $(\alpha, \kappa)$ parameter plane. Crude as this plot is, its message is still clear. The stable chimera state evidently lives in a wedge in parameter space, bounded on one side by the line $\alpha=\frac{\pi}{2}$ and on the other by a curve $\alpha=\alpha_{c}(\kappa)$ that is nearly a straight line. By its very shape, the picture directs our attention to the corner of the wedge, to the simultaneous limit as $\alpha \rightarrow \frac{\pi}{2}$ from below and $\kappa \rightarrow 0$ from above. Apparently something crucial happens in that corner-the chimera state is born there. And so this is where perturbation theory should be conducted. 


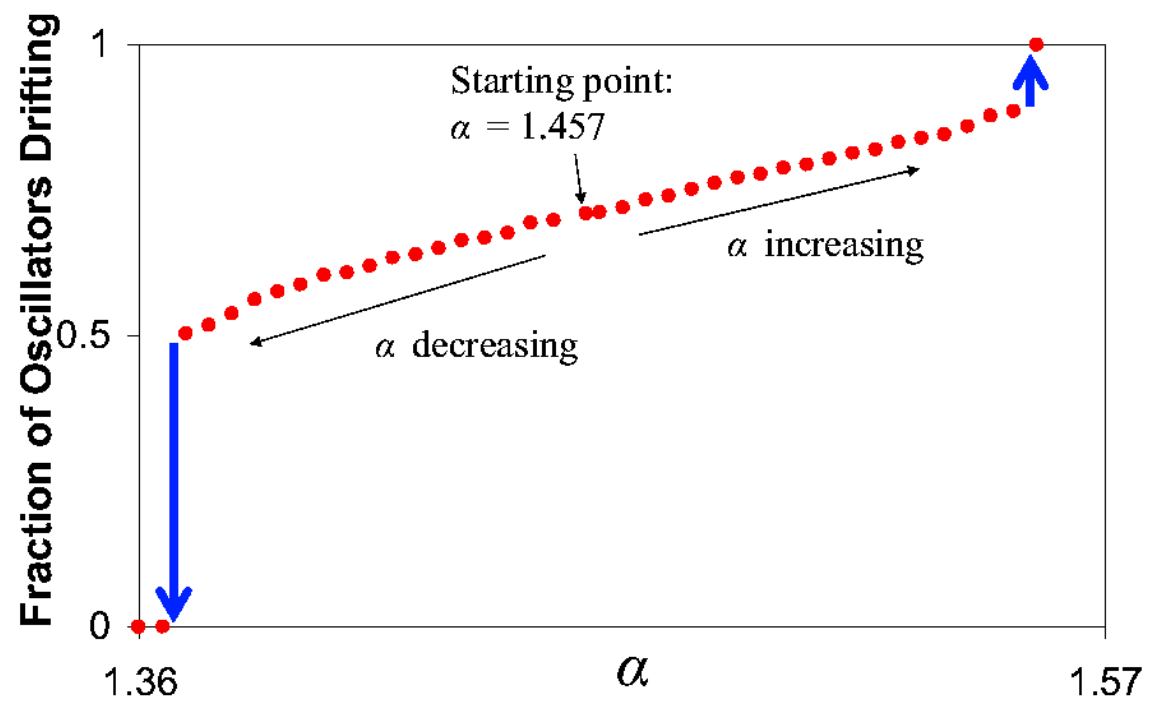

FIG. 4: The fraction of oscillators drifting as the coupling parameter $\alpha$ varies. Here $\kappa=4.0$, $N=256$ oscillators, $d t=0.025$ for 100,000 iterations.

To check that the wedge of Fig. 5 was not an artifact of the exponential kernel assumed above, we also calculated the corresponding contour plots for the cosine kernel

$$
G(x)=\frac{1}{2 \pi}(1+A \cos x),
$$

where $0 \leq A \leq 1$. Here the spatial domain has been redefined to $-\pi \leq x \leq \pi$ for convenience, and to bring out its ring geometry and the reflection symmetry of the chimera state. Figure 6] confirms that the cosine kernel gives a similar chimera state to that for the exponential kernel used above, while Fig. [7] demonstrates that the wedge in parameter space is preserved as well. All that is reassuring, because as it happens, the cosine kernel also has the pleasant property that it allows the self-consistency equation to be solved analytically.

\section{AN EXACTLY SOLVABLE CASE}

From now on, let $G(x)$ be given by the cosine kernel (11), and let the spatial domain be $-\pi \leq x \leq \pi$ with periodic boundary conditions. For this case, we'll show that the functional form of the order parameter can be obtained exactly, which in turn yields the explicit $x$-dependence of $R(x)$ and $\Theta(x)$. All the resulting expressions, however, still contain two unknown coefficients, one real and the other complex, which need to be determined selfconsistently. In this way, the two unknown functions in the self-consistency equation are 


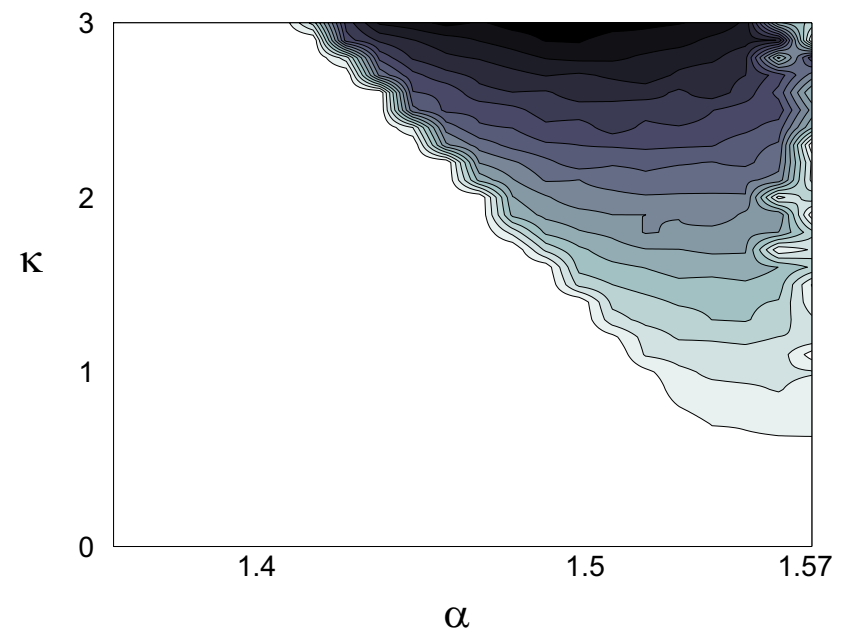

FIG. 5: Amplitude of the curve $R(x)$, depicted as a contour plot in parameter space, and calculated by averaging over the instantaneous $R$ curves during numerical integration. Here $G(x) \propto \exp (-\kappa|x|), N=80$ oscillators, the integration time step is $d t=0.025$, and integration continued for 20,000 iterations. Lighter colors indicate smaller amplitude; lightest is $R_{a m p}=0.0$ and darkest is $R_{a m p}=0.13$.

exchanged for two unknown numbers - a drastic reduction in the difficulty of the problem.

The self-consistency equation (10) is

$$
R(x) e^{i \Theta(x)}=e^{i \beta} \int_{-\pi}^{\pi} G\left(x-x^{\prime}\right) e^{i \Theta\left(x^{\prime}\right)} h\left(x^{\prime}\right) d x^{\prime}
$$

where we've introduced the notation

$$
h\left(x^{\prime}\right)=\frac{\Delta-\sqrt{\Delta^{2}-R^{2}\left(x^{\prime}\right)}}{R\left(x^{\prime}\right)} .
$$

Let angular brackets denote a spatial average:

$$
\langle f\rangle=\frac{1}{2 \pi} \int_{-\pi}^{\pi} f\left(x^{\prime}\right) d x^{\prime} .
$$

Then, substituting the cosine kernel (11) into (12) and expanding $G\left(x-x^{\prime}\right)$ by a trigonometric identity, we find

$$
\begin{aligned}
R e^{i \Theta} & =\frac{e^{i \beta}}{2 \pi} \int_{-\pi}^{\pi}\left[1+A \cos x \cos x^{\prime}+A \sin x \sin x^{\prime}\right] h\left(x^{\prime}\right) e^{i \Theta\left(x^{\prime}\right)} d x^{\prime} \\
& =e^{i \beta}\left\langle h e^{i \Theta}\right\rangle+e^{i \beta} A\left\langle h e^{i \Theta} \cos x^{\prime}\right\rangle \cos x+e^{i \beta} A\left\langle h e^{i \Theta} \sin x^{\prime}\right\rangle \sin x \\
& =c+a \cos x
\end{aligned}
$$




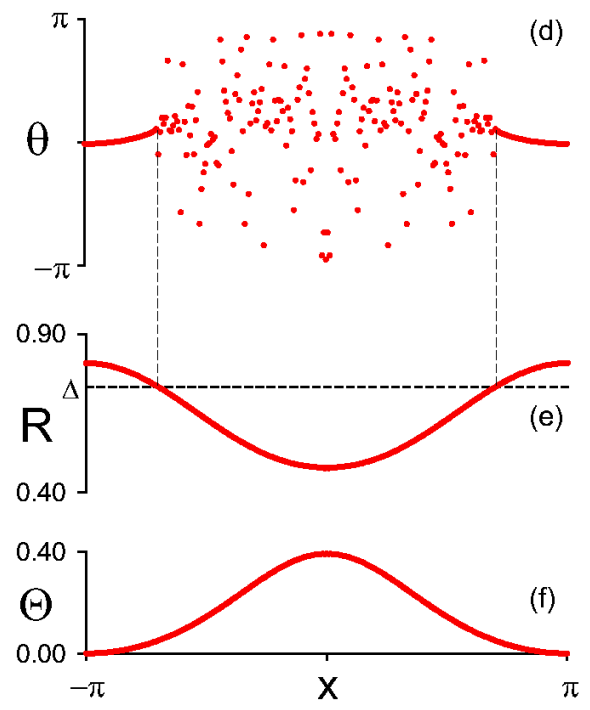

FIG. 6: Chimera state and corresponding order parameter curves for the cosine kernel, shown in the same format as Fig. 3. and qualitatively similar to it. Parameters are $A=0.995, \beta=0.18$, $N=256$ oscillators; equation (10) was integrated with fixed time step $d t=0.025$ for 200,000 iterations, starting from $\phi(x)=6 r \exp \left(-0.76 x^{2}\right)$, where $r$ is a uniform random variable on $\left[-\frac{1}{2}, \frac{1}{2}\right]$.

where the coefficients $c$ and $a$ must satisfy their own version of the self-consistency equations, now given by

$$
c=e^{i \beta}\left\langle h e^{i \Theta}\right\rangle
$$

and

$$
a=A e^{i \beta}\left\langle h e^{i \Theta} \cos x^{\prime}\right\rangle
$$

Note that the coefficient of $\sin x$ vanishes in (14). This follows from the assumption that $R\left(x^{\prime}\right)=R\left(-x^{\prime}\right)$ and $\Theta\left(x^{\prime}\right)=\Theta\left(-x^{\prime}\right)$, as suggested by the simulations; then $h\left(x^{\prime}\right)$ in (13) is also even, and so the integral $\left\langle h e^{i \Theta} \sin x^{\prime}\right\rangle$ in (14) vanishes by oddness. As we'll show next, this assumption of reflection symmetry is self-consistent, in the sense that it implies formulas for $R(x)$ and $\Theta(x)$ that indeed possess this symmetry.

For example, to calculate $R(x)$ in terms of the unknown coefficients $a$ and $c$, observe that

$$
\begin{aligned}
R^{2} & =\left(R e^{i \Theta}\right)\left(R e^{-i \Theta}\right) \\
& =(c+a \cos x)\left(c^{*}+a^{*} \cos x\right) \\
& =\left|c^{2}\right|+2 \operatorname{Re}\left(c a^{*}\right) \cos x+|a|^{2} \cos ^{2} x
\end{aligned}
$$

which is an even function, and which also helps to explain why the graph of $R(x)$ in Fig. 3 


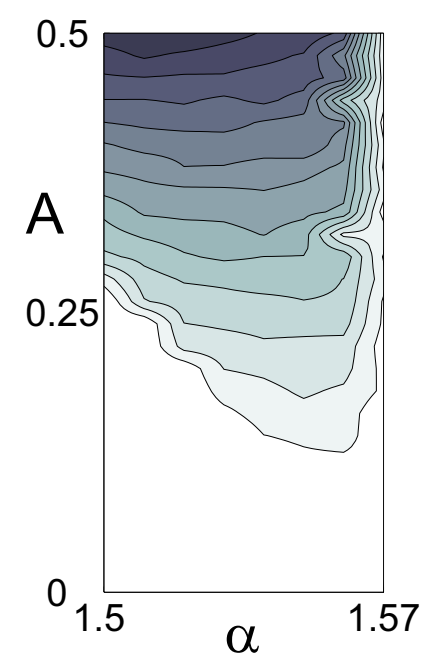

FIG. 7: Contour plot of $R_{a m p}$ for the chimera state with cosine kernel. Note the similarity to Fig. 5 for the exponential kernel. Here $G(x)=\frac{1}{2 \pi}(1+A \cos x), N=80$ oscillators, the integration time step was $d t=0.025$, and integration continued for 30,000 iterations. Same color scale as Fig. [5].

resembled a cosine wave.

Likewise, $\Theta(x)$ is an even function reminiscent of a cosine because

$$
\begin{aligned}
\tan \Theta(x) & =\frac{R(x) \sin \Theta(x)}{R(x) \cos \Theta(x)} \\
& =\frac{\operatorname{Im}(c)+\operatorname{Im}(a) \cos x}{\operatorname{Re}(c)+\operatorname{Re}(a) \cos x} .
\end{aligned}
$$

Another simplification is that $c$ can be taken to be purely real and non-negative, without loss of generality. This follows from the rotational symmetry of the governing equations. In particular, the self-consistency equation (12) is left unchanged by any rigid rotation $\Theta(x) \rightarrow \Theta(x)+\Theta_{0}$. Thus we are free to specify any value of $\Theta(x)$ at whatever point we like. The most convenient choice is to set

$$
\Theta\left(\frac{\pi}{2}\right)=0
$$

Then at that value of $x$ the equation $R e^{i \Theta}=c+a \cos x$ reduces to

$$
R\left(\frac{\pi}{2}\right)=c
$$

Since $R$ is real and non-negative, so is $c$. Hence, we take

$$
\operatorname{Im}(c)=0
$$


from now on.

The final step in closing the equations for $a$ and $c$ is to rewrite the averages in (15) and (16) in terms of those variables. To do so, we express $h e^{i \Theta}$ as

$$
\begin{aligned}
h e^{i \Theta} & =\left(R e^{i \Theta}\right) \frac{h}{R} \\
& =(c+a \cos x) \frac{\Delta-\sqrt{\Delta^{2}-R^{2}(x)}}{R^{2}(x)} \\
& =\frac{\Delta-\sqrt{\Delta^{2}-R^{2}(x)}}{c+a^{*} \cos x}
\end{aligned}
$$

where we have used (17) and the real-valuedness of $c$ to simplify the second line above. Inserting (17) and (20) into (15) and (16), we obtain the desired self-consistency equations for $a$ and $c$ :

$$
\begin{gathered}
c=e^{i \beta}\left\langle\frac{\Delta-\left(\Delta^{2}-c^{2}-2 \operatorname{Re}(a) c \cos x-|a|^{2} \cos ^{2} x\right)^{\frac{1}{2}}}{c+a^{*} \cos x}\right\rangle \\
a=A e^{i \beta}\left\langle\frac{\Delta-\left(\Delta^{2}-c^{2}-2 \operatorname{Re}(a) c \cos x-|a|^{2} \cos ^{2} x\right)^{\frac{1}{2}}}{c+a^{*} \cos x} \cos x\right\rangle .
\end{gathered}
$$

This pair of complex equations is equivalent to four real equations for the four real unknowns $c, \operatorname{Re}(a), \operatorname{Im}(a)$, and $\Delta$. The solutions, if they exist, are to be expressed as functions of the parameters $\beta$ and $A$.

\section{CLUES BASED ON NUMERICS}

Before we plunge into the details of solving equations (21) and (22) simultaneously, let's pause to remember what we're trying to do.

We want to understand where the chimera state lives in parameter space and how it bifurcates. Guided by the simulations of Section [II] we expect that (21), (22) should have chimera solutions throughout the wedge-shaped region of parameter space shown in Fig. [7. Assuming that's true, we hope that these solutions will continue all the way down to the

corner $(\alpha, A)=\left(\frac{\pi}{2}, 0\right)$, corresponding to $(\beta, A)=(0,0)$, where might be able to analyze them with perturbation theory.

Our strategy, then, is to start by finding one solution to (21), (22), by any means possible, for parameter values anywhere in the wedge. Having found this solution, we can use it as a base point for a numerical continuation method. Then we proceed to dive into the corner, 
following a straight line through parameter space between the base point and the corner. In this way we convert the problem to a one-parameter study of the solutions of (21), (22). Sufficiently close to the corner, we expect that the solutions will display some sort of scaling behavior with respect to the parameter. That scaling will then suggest clues about the right ansatz for a subsequent perturbation calculation.

So first we have to come up with a chimera solution to (21), (22). It's not just a matter of plugging the equations into a standard root-finding package. The trouble is that these equations also have other solutions that we're less interested in, and we don't want the numerical root-finding scheme to converge to them instead.

In particular, the in-phase solution, where all the oscillators are locked at the same phase and none of them are drifting, has a large basin of attraction that competes with that of the chimera state. To see what values of $a, c$, and $\Delta$ correspond to the in-phase state, note that when $\phi(x, t)=\phi\left(x^{\prime}, t\right)$ for all $x$ and $x^{\prime}$, Eq. (II) implies $\phi(x, t)=\phi_{0}+(\omega-\sin \alpha) t$. Hence $R=1$ and therefore $c=1$ and $a=0$. And because $\Omega=\omega-\sin \alpha=\omega-\cos \beta$, we have $\Delta=\omega-\Omega=\cos \beta$. Thus

$$
(a, c, \Delta)_{\text {in-phase }}=(0,1, \cos \beta)
$$

It's easy to check that this satisfies (21), (22) for all values of $A$ and $\beta$.

To reduce the chance that the root-finder will converge onto this in-phase state, we need to concoct an initial guess that's very close to a genuine chimera state. To find one, we numerically integrated Eq. (11) using the cosine kernel, and fit the resulting graphs of $R(x)$ and $\Theta(x)$ to the exact formulas (17) and (18), to estimate the values of $a$ and $c$. The frequency difference $\Delta$ was obtained directly from the simulation, by setting $\omega=0$ and then computing $\Delta=\omega-\Omega=-\Omega$, where $\Omega$ is observable as the collective frequency of the locked oscillators.

In this way we estimated $a=0.156-0.072 i, c=0.591, \Delta=0.720$ for the stable chimera state at parameter values $A=0.99, \beta=0.081$. We fed this starting guess into the Matlab root-finding and numerical continuation program MatCont [5] and found rapid convergence to $a=0.162-0.051 i, c=0.588, \Delta=0.723$. From there, we could continue the solution in either $A$ or $\beta$ or some combination, as we saw fit.

This approach enabled us to track the chimera state throughout parameter space, until it disappeared along a critical curve corresponding to the boundary of the wedge shown 


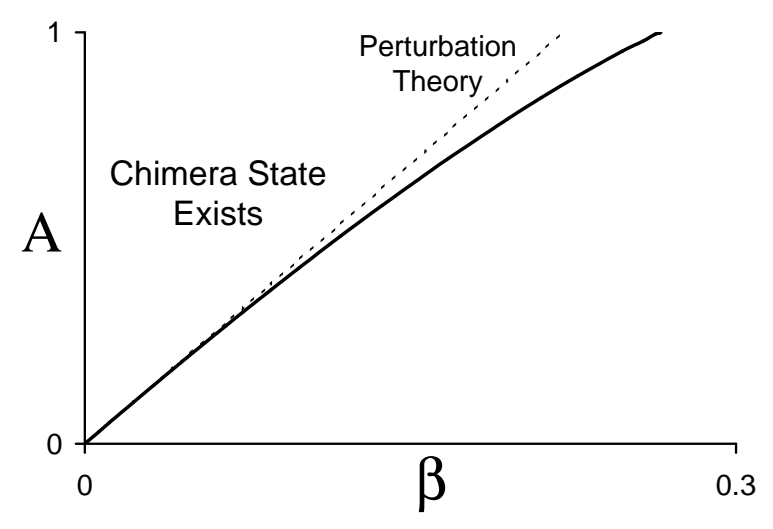

FIG. 8: The region of parameter space in which the chimera state exists. Solid line, exact boundary determined by numerical solution of (21) and (22); dashed line, leading order approximation to this boundary obtained by perturbation theory (see text).

earlier. The results of this calculation are shown in Fig. 8. As expected, the boundary of the region is nearly a straight line, and it extends down to the origin.

\section{PERTURBATION THEORY}

The next step is to look for scaling laws to guide our perturbation calculations. Figure 9 shows the results of numerical continuation starting from $(\beta, A)=(0.08,0.99)$ and moving along the line $A=12.375 \beta$ towards the origin, all the while remaining within the wedge shown in Fig. 8. The observed behavior of the variables along that line suggests the following ansatz near the origin:

$$
\begin{aligned}
\Delta & \sim 1+\Delta_{1} \epsilon+\Delta_{2} \epsilon^{2} \\
c & \sim 1+c_{1} \epsilon+c_{2} \epsilon^{2} \\
\operatorname{Re}(a) & \sim u \epsilon^{2} \\
\operatorname{Im}(a) & \sim v \epsilon^{2}
\end{aligned}
$$

where we have introduced $\epsilon=A$ as the small parameter.

Next, we assume that this ansatz continues to hold along other lines through the origin. Such lines can be parametrized as

$$
\begin{gathered}
A=\epsilon, \\
\beta=\beta_{1} \epsilon,
\end{gathered}
$$



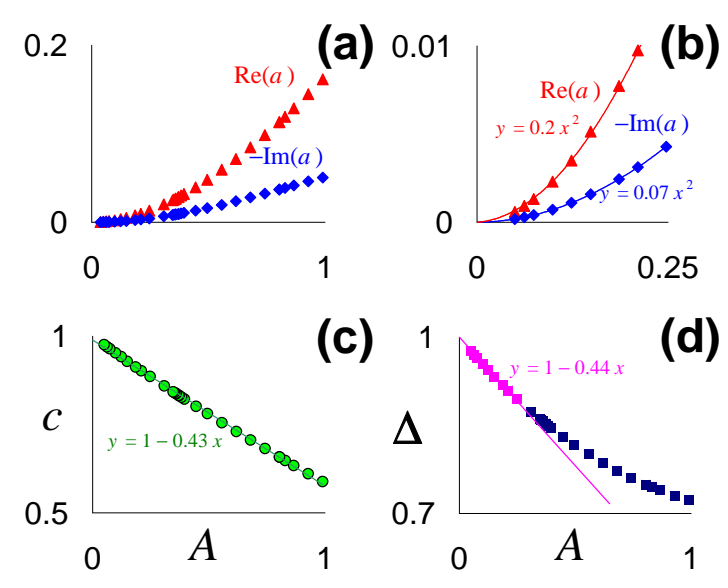

FIG. 9: Scaling laws near the origin in parameter space, along the line $A=12.375 \beta$. Data were collected from numerical continuation of a known chimera state, for an ensemble of parameter values. Approximate fits were then determined by least-square regression. (a) Scaling of real and imaginary parts of $a$; (b) Zoom of panel (a) near origin in parameter space. Note that curves are quadratic; (c) Linear scaling of real-valued variable $c$; (d) Scaling of $\Delta$. Note that $\Delta$ scales linearly for small values of $A$ (purple).

where $A$ and $\beta$ tend to zero simultaneously as $\epsilon \rightarrow 0$. Here $\beta_{1}$ is a free parameter inversely related to the slope of the lines. Thus the asymptotic shape of the wedge in Fig. 8, sufficiently close to the origin, will be determined from the maximum and minimum values of $\beta_{1}$ for which a perturbative solution exists.

Substituting the ansatz (24) into the self-consistency equation (21) for $c$, and retaining only terms up to $\mathcal{O}(\sqrt{\epsilon})$ gives

$$
\begin{aligned}
1+\mathcal{O}(\epsilon) & =\left(1+i \beta_{1} \epsilon\right)\left\langle\frac{1+\Delta_{1} \epsilon-\left(1+2 \Delta_{1} \epsilon-1-2 c_{1} \epsilon\right)^{\frac{1}{2}}}{1+c_{1} \epsilon}\right\rangle \\
& =1-\sqrt{2} \sqrt{\Delta_{1}-c_{1}} \sqrt{\epsilon}+\mathcal{O}(\epsilon)
\end{aligned}
$$

implying that

$$
\Delta_{1}=c_{1}
$$

Now we retain terms up to $\mathcal{O}(\epsilon)$ on both sides, and apply Eq. (25) whenever necessary to cancel terms. At this order, Eq. (21) becomes

$$
1+c_{1} \epsilon=1+i \beta_{1} \epsilon-\epsilon \sqrt{2}\left\langle\sqrt{\left(\Delta_{2}-c_{2}\right)-u \cos x}\right\rangle
$$


To simplify notation, let

$$
\delta=\Delta_{2}-c_{2}
$$

After breaking up the previous expression (26) into two equations for the real and imaginary parts, and equating terms of $\mathcal{O}(\epsilon)$, we get

$$
\begin{aligned}
& c_{1}=-\operatorname{Re}[\sqrt{2}\langle\sqrt{\delta-u \cos x}\rangle] \\
& \beta_{1}=\operatorname{Im}[\sqrt{2}\langle\sqrt{\delta-u \cos x}\rangle] .
\end{aligned}
$$

Repeating the same expansion to $\mathcal{O}(\epsilon)$ in the self-consistency equation (22) for $a$ yields two analogous equations:

$$
\begin{aligned}
& u=-\operatorname{Re}[\sqrt{2}\langle\cos x \sqrt{\delta-u \cos x}\rangle] \\
& v=-\operatorname{Im}[\sqrt{2}\langle\cos x \sqrt{\delta-u \cos x}\rangle] .
\end{aligned}
$$

The equations (28)-(31) form a closed system for the variables $\left(c_{1}, u, v, \delta\right)$, given the parameter $\beta_{1}$. But to solve these equations, it proves more convenient to regard $\beta_{1}$ as a variable, and $\delta$ as a parameter; we adopt this point of view in what follows.

There's another important structural aspect of equations (28)-(31), namely, that (30) is distinguished in that it involves only two unknown quantities. It has the form $u=f(u, \delta)$ and can be solved numerically for a given $\delta$. When a solution exists, all other variables $\left(c_{1}\right.$, $\left.\beta_{1}, v\right)$ can be generated parametrically from the $(u, \delta)$ pair. Thus, the problem of solving equations (28)-(31) reduces to a root-finding exercise in one dimension instead of four.

Figure 10 plots the graph of the difference $f(u, \delta)-u$ for several values of $\delta$. The zeros of this graph correspond to the solutions of (30), and yield the desired $(u(\delta), \delta)$ pairs. These are then substituted into the remaining equations to obtain $c_{1}(\delta), \beta_{1}(\delta)$, and $v(\delta)$, from which various quantities of physical interest can be derived.

\section{A. Calculation of $f_{\text {drift }}$}

For example, we can use the perturbative solution to find $f_{\text {drift }}$, the fraction of the system that is drifting. It is most convenient to calculate this quantity first in terms of $\delta$, and then later re-express it in terms of the more natural control parameter $\beta_{1}$.

To find the drifting oscillators, recall from Fig. 6] that the cutoff between the locked portion and the drifting portion occurs at the crossover value $x=x_{c}$ where $R\left(x_{c}\right)=|\Delta|$. 


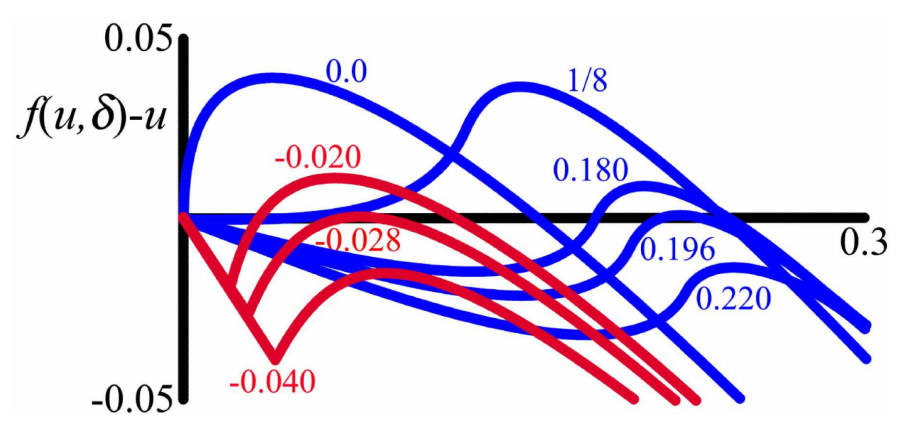

FIG. 10: Roots of Eq. (30) for various values of $\delta$. Red indicates negative $\delta$ and blue positive $\delta$. For $\delta<-0.028$ there are no roots; for $-0.028<\delta<0$, two roots; for $0<\delta<\frac{1}{8}$, one root; for $\frac{1}{8}<\delta<0.196$, two roots; and for $\delta>0.196$, no roots .

Substituting (17) for $R^{2}$ and equating this to $\Delta^{2}$, we obtain

$$
|c|^{2}+2 \operatorname{Re}\left(c a^{*}\right) \cos x_{c}+|a|^{2} \cos ^{2} x_{c}=\Delta^{2} .
$$

Plugging in the ansatz (24) and keeping terms up to order $\epsilon^{2}$, we find

$$
1+2 c_{1} \epsilon+\left(c_{1}^{2}+2 c_{2}\right) \epsilon^{2}+2 u \epsilon^{2} \cos x_{c}=1+2 \Delta_{1} \epsilon+\left(\Delta_{1}^{2}+2 \Delta_{2}\right) \epsilon^{2} .
$$

Finally, because of (25), this simplifies to

$$
\cos x_{c}=\frac{\Delta_{2}-c_{2}}{u}=\frac{\delta}{u}
$$

Since the spatial domain of the ring has length $2 \pi$ and $2 x_{c}$ is the length of the region occupied by drifting oscillators, the fraction of the chimera state corresponding to drifting oscillators is $f_{\text {drift }}=x_{c} / \pi$, and hence

$$
f_{\text {drift }}=\frac{1}{\pi}\left|\cos ^{-1}\left(\frac{\delta}{u(\delta)}\right)\right| .
$$

Figure[11 plots the numerically computed $f_{\text {drift }}(\delta)$ against $\beta_{1}(\delta)$. The curve has a turning point at $\beta_{1} \approx 0.22$, when about $44 \%$ of the system is drifting. Presumably, this turning point stems from a saddle-node bifurcation in the underlying dynamics. In our simulations, we only see the upper branch of this curve, suggesting that this corresponds to the stable version of the chimera state. The reciprocal of the critical $\beta_{1}$ is about 4.5 , which is the slope of the dashed line shown in Fig. 8, in excellent agreement with the boundary of the wedge found numerically. 


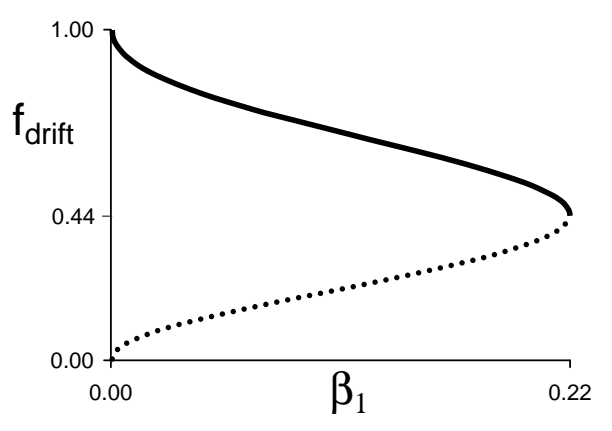

FIG. 11: Fraction of chimera state consisting of drifting oscillators as a function of $\beta_{1}$. Solid line indicates stable chimera, dotted line indicates unstable. The maximum $\beta_{1}$ determines the line bounding the wedge-shaped existence region in Fig. 8

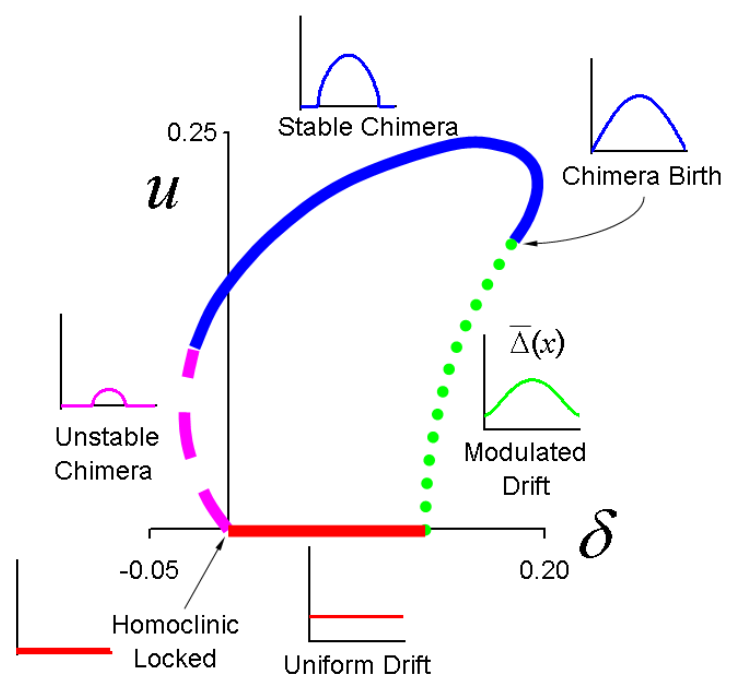

FIG. 12: Diagram of bifurcations giving rise to the chimera state in $u-\delta$ plane. Insets show average frequency $\bar{\Delta}$ versus $x$. Please see text for definitions of the perturbative variables $\delta$ and $u$, and for a detailed explanation of this figure.

\section{B. Birth and Death of the Chimera State}

Although the parametric dependence of $f_{\text {drift }}$ seems to be conveniently expressed with respect to $\beta_{1}$, that representation conceals a lot. Several dynamically distinct states of the system are invisible because they are all squeezed onto a single point $\left(\beta_{1}, f_{\text {drift }}\right)=(0,1)$, as we'll see below. It's much more revealing to use $\delta$ instead of $\beta_{1}$.

Therefore, we now examine the system in the set of coordinates shown in Fig. 12, with 
$u$ plotted vertically and $\delta$ horizontally. This picture is a compendium of all the stationary states of the system - the stable and unstable chimera, along with other states that we haven't mentioned yet, which we call uniform drift, modulated drift, and homoclinic locked states. The virtue of this representation is that it allows us to see each bifurcation that occurs as the chimera state comes into existence and later disappears. Beginning at the origin and moving counterclockwise around the kidney-bean shaped cycle, we have:

1. Homoclinic locked state: $u=\delta=0$. Here, all the oscillators are locked in phase, and hence frozen in the rotating frame. Accordingly, the average frequency $\bar{\Delta}(x)$ of the oscillators vanishes for all $x$, as shown in the inset. But one can show that this state is not linearly stable. In fact, the exact, non-perturbative counterpart of this state is the in-phase locked state (23) at the critical parameter value $\beta=0$, where this state undergoes a homoclinic saddle-node bifurcation.

2. Spatially uniform drift: For $u=0$ and small $\delta>0$, the system has a stationary state in which all the oscillators drift in a way that varies strongly in time but remains uniform in $x$. The order parameter $R(x)$ is independent of $x$ and close to 1 , meaning that the oscillators are nearly in phase for nearly all of the time. An individual oscillator executes a jerky motion around its phase circle, lingering near $\theta=0$ and then whipping around the rest of the phase circle back to this point. The associated plot of $\bar{\Delta}(x)$ is flat because of the uniformity in $x$.

In terms of the perturbative variables used in Fig. 12, this state appears on the line $u=0$ with $\delta>0$. Then (131) shows that $v=0$ as well; hence $a=0$, to $\mathcal{O}\left(\epsilon^{2}\right)$. So (17) implies that $R(x)$ must be real and constant and (18) implies that $\Theta(x)=0$. Equation (29) tells us that such a state is possible only if $\beta_{1}=0$, which suggests that one can find an exact, non-perturbative version of the uniform drift state when $\beta=0$. Indeed, seeking a solution of the self-consistency equation (12) with $\beta=0, \Theta(x)=0$, and constant $R(x)$, one finds $R^{2}=\Delta-\sqrt{\Delta^{2}-R^{2}}$ since the kernel $G$ is normalized. Hence, along this line $\beta=0, \Theta(x)=0$, and $R(x)=R=\sqrt{2 \Delta-1}$.

3. Onset of spatial structure: At the lower right corner of the kidney bean, the function $f(u, \delta)-u$ (Fig. 10) becomes tangent at the origin, introducing a new branch of solutions with $\Theta=0$ and $\beta=0$ but with the coherence $R$ varying spatially. This is 
the birth of spatial structure in the system. It happens for $\delta=\frac{1}{8}$. The non-perturbative

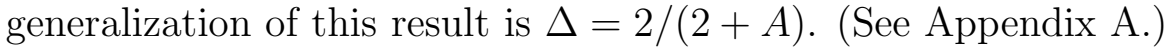

4. Modulated drift: Along the first curved branch, all oscillators continue to drift, but now there is spatial structure in the $R(x)$ curve, leading to a modulated pattern of average velocities (see inset). However, the average angle $\Theta(x)$ is still identically 0 .

5. Chimera birth: At the point where $u=\delta$, the first locked oscillators are born. For the first time, $v$ and $\beta_{1}$ become nonzero (see Eq. (29),(31)). Until this point, all of the states have been confined to the vertical axis of Fig. 8 now we finally we move off the wall. The curve of average velocities $\bar{\Delta}(x)$ touches the $x$-axis at a single point. Meanwhile, the system develops spatial structure in its average phase: $\Theta(x)$ is no longer identically zero.

This bifurcation can be shown to occur at $\delta=16 /\left(9 \pi^{2}\right)$, by evaluating the integral in (301) with $u=\delta$; also, see Appendix B for an exact calculation of the chimera state at birth.

6. Stable chimera: Along the top of the kidney bean, the chimera state is dynamically stable. After its birth from the spatially modulated drift state, it gradually develops an increasing fraction of locked oscillators as we move in the counterclockwise direction. Locked oscillators correspond to the zero part of the $\bar{\Delta}(x)$ curve (they appear motionless because the reference frame was chosen to co-rotate with them).

7. Saddle-node bifurcation: As we continue to move counterclockwise, the value of $\beta_{1}$ grows (along with the fraction of locked oscillators), and reaches its maximum at the point where the stable chimera ceases to exist. The disappearance is a result of a saddle-node bifurcation - a collision with an unstable chimera state - and occurs when about $44 \%$ of the system is drifting.

8. Unstable chimera: Along the unstable dashed branch, the fraction of locked oscillators continues to grow. But the value of $\beta_{1}$ now begins to decrease, indicating a movement back towards the wall in Fig. 8 . The system returns to its original state when all oscillators become locked, with $\delta=u=\beta_{1}=0$ and $R=1$. 
Taking a step back, we can see an interesting message of Fig. 12. The stable and unstable chimera states are continuously connected though the branches of drifting states, shown in solid red and dotted green lines. If we had used the $\beta_{1}$ representation instead (as in Fig. (11), both of these connecting branches would have shrivelled down to a point. The two kinds of chimera states would seem disconnected in a way that they really aren't.

\section{DISCUSSION}

Our main result in this paper is an exact solution for the chimera state, for the special case of a cosine kernel. That solution also shed light on the bifurcations which create and destroy the chimera.

In retrospect, it's not surprising that a cosine kernel would make the self-consistency equation (10) as tractable as possible, because the right hand side of (10) is a convolution integral, and trigonometric functions behave nicely under convolution. For this reason, it should be straightforward to extend the calculations to include more harmonics in $G$. Using the same argument as in Section IV] one can see that the exact solution for the order parameter (14) will have the same number of harmonics as $G$ has. This approach would then give a systematic way to solve the self-consistency equation for any kernel representable as a finite Fourier cosine series. By taking more and more terms, this approach also gives a way to approximate results for any even kernel, as long as it is representable by a Fourier series.

Unfortunately, the trick of choosing a special kernel may not work as well in two (or three) spatial dimensions. That could limit the applicability to two-dimensional chimeras, such as the novel spiral waves computed numerically in [10]. Nevertheless, the idea of seeking a tractable kernel that can simplify the problem may itself be useful.

Another caveat is that, despite its usefulness as a mathematical tool, the perturbative approach adopted here does not give a rigorous understanding of the bifurcations in the original problem. One would like to understand the bifurcation scenarios for all values of the coupling parameter $A$, which essentially measures how far the nonlocal coupling deviates from strictly global coupling. In Appendices $\mathrm{A}$ and $\mathrm{B}$, we show two results along these lines.

One interesting aspect of the perturbative approach is that it draws our attention to the special parameter values $A=0, \beta=0$ (or equivalently $\alpha=\pi / 2$ ). Here the system has 
global cosine coupling and is known to be completely integrable [23, 24]. So in a sense, what we have done in this paper is perturb off this extremely degenerate system, which raises the question of whether other, unforeseen attractors might also lurk nearby, for different choices of initial conditions.

The surprising nature of the chimera state makes us wonder if it could be created artificially in a laboratory experiment, or possibly even occur naturally in some system.

As a first attempt to judge whether this might be possible, we tried to integrate the phase equation (11) with slightly nonuniform frequencies $\omega_{i}$, to mimic the inhomogeneities that would occur in any real system, and to test whether the chimera is an artifact of assuming identical oscillators. We added a uniform random variable $r \in[-B, B]$ to the native frequency $\omega$ for each oscillator, and we found the chimera state persisted, as long as $B$ was not too large - less than about $4 \%$ of $\Delta$ (the frequency difference between the locked oscillators' $\Omega$ and the mean natural frequency $\omega$ ). This estimate should be conservative when compared with a presumably more realistic Gaussian random distribution of $\omega_{i}$.

There are several possibilities for experimental systems where the distinctive effects of nonlocal coupling, including the chimera state, might be observed. Laser arrays seem to be good candidates. In some cases, such as semiconductor arrays with evanescent coupling [14, 28], they are governed by equations similar to (1), though these are usually approximated as nearest-neighbor. Likewise, phase equations of this form arise in the description of coupled electronic phase-locked loops, and superconducting arrays of Josephson junctions [20, 25]. Finally, an idealized model of biochemical oscillators, coupled by a diffusible substance that they all produce, can give rise to an effectively nonlocal coupling and chimera states; indeed, this was the motivating example that led Kuramoto and his colleagues to their discovery.

Whether or not the chimera state turns out to experimentally realizable, it is fascinating in its own right, as a strange new mechanism for pattern formation in spatially extended nonlinear systems. Its existence underscores how much still remains to be discovered, even in what would seem to be the simplest possible model of pattern formation: a one-dimensional collection of identical oscillators. 


\section{ACKNOWLEDGMENTS}

Research supported in part by the National Science Foundation. We thank Yoshiki Kuramoto for helpful correspondence, Steve Vavasis for advice about solving the self-consistency equation numerically, Bard Ermentrout for drawing our attention to bump states in neural systems, Dan Wiley and Herbert Hui for helpful discussions, and Kim Sneppen and the Niels Bohr Institute for being such gracious hosts. 


\section{APPENDIX A: ONSET OF SPATIAL STRUCTURE}

We now show that the birth of spatial structure can be calculated non-perturbatively. We have already seen in Section VIB that when $\beta=0$, the system has an exact state of spatially uniform drift with constant coherence $R(x) \equiv \sqrt{2 \Delta-1}$ and average phase $\Theta(x) \equiv 0$. For this special state, the modulation amplitude $a=0$ and the mean level of the coherence $c=R=\sqrt{2 \Delta-1}$. At the bifurcation that creates spatial variation in the coherence, the real part of a becomes nonzero; at leading order in perturbation theory, this bifurcation

takes place at $\delta=\frac{1}{8}$. Meanwhile, the imaginary part of a remains zero, which means that $\Theta(x) \equiv 0$ still holds.

To generalize this result to the non-perturbative case, we seek conditions for a second branch of solutions to bifurcate off the uniform drift state. Since $\Delta=\left(1+c^{2}\right) / 2$ for the drift state, we consider a slight perturbation

$$
\Delta=\left(1+c^{2}\right) / 2+\eta
$$

where $\eta$ is a small deviation. Also, since $a=0$ for the uniform drift state, we may take $a$ itself as a small deviation. Plugging all this into the self-consistency equation (21) gives:

$$
c=\left\langle\frac{\left(1+c^{2}\right) / 2+\eta-\left[\left(\left(1+c^{2}\right) / 2+\eta\right)^{2}-c^{2}-2 a c \cos x-a^{2} \cos ^{2} x\right]^{\frac{1}{2}}}{c+a \cos x}\right\rangle .
$$

Now expand in a two-variable Taylor series for small $\eta$ and $a$, and integrate over $x$ to obtain:

$0=\left[\frac{2 c}{c^{2}-1} \eta+\mathcal{O}\left(\eta^{2}\right)\right]+\left[\frac{c\left(c^{2}-3\right)\left(c^{2}+1\right)}{2\left(1-c^{2}\right)^{3}}+\frac{c\left(c^{6}-5 c^{4}+19 c^{2}+9\right)}{\left(1-c^{2}\right)^{5}} \eta+\mathcal{O}\left(\eta^{2}\right)\right] a^{2}+\mathcal{O}\left(a^{4}\right)$.

Repeating the approach for the second self-consistency equation (22) gives:

$$
0=\left[\frac{2\left(c^{2}-1\right)+A\left(c^{2}+1\right)}{2\left(c^{2}-1\right)}+\frac{A\left(c^{4}-4 c^{2}-1\right)}{\left(c^{2}-1\right)^{3}} \eta+\mathcal{O}\left(\eta^{2}\right)\right] a+\mathcal{O}\left(a^{3}\right) .
$$

To locate where another branch of solutions bifurcates off the uniform drift solution, we inspect the linearization of the algebraic system above, given by the Jacobian matrix

$$
\left[\begin{array}{cc}
\frac{2 c}{\left(c^{2}-1\right)^{2}} & 0 \\
0 & \frac{2\left(c^{2}-1\right)+A\left(c^{2}+1\right)}{2\left(c^{2}-1\right)}
\end{array}\right]
$$

If the determinant of the Jacobian is nonzero, the implicit function theorem tells us that no other solutions exist nearby. Hence, the existence of a continuously bifurcating branch 
requires that the determinant vanish. Setting the determinant equal to zero yields $c=0$ or $2\left(c^{2}-1\right)+A\left(c^{2}+1\right)=0$. Plugging in the value of $c$ about which we're linearizing, $c=\sqrt{2 \Delta-1}$, and solving for $\Delta$ finally gives the bifurcation condition

$$
\Delta_{c}=\frac{2}{A+2} .
$$

To compare this with our earlier result from first-order perturbation theory, we express the perturbative variable $\delta$ at this critical point by using its definition from (27) above and the property in (25). Since $\Delta-c=\delta \epsilon^{2}=\delta A^{2}$ (ignoring higher order terms), we have $\delta=\frac{\Delta-c}{A^{2}}$. So

$$
\begin{aligned}
\delta_{c} & =\frac{\Delta_{c}-c}{A^{2}} \\
& =\frac{1}{A^{2}}\left(\frac{2}{A+2}-\sqrt{2 \Delta_{c}-1}\right) \\
& =\frac{1}{A^{2}}\left(\frac{2}{A+2}-\sqrt{\frac{2-A}{2+A}}\right) \\
& =\frac{1}{8}-\frac{1}{16} A+\frac{5}{128} A^{2}-\frac{5}{256} A^{3}+\mathcal{O}\left(A^{4}\right),
\end{aligned}
$$

which agrees with our perturbative prediction that $\delta_{c}=\frac{1}{8}$ in the limit that $A \rightarrow 0$. 


\section{APPENDIX B: BIRTH OF THE CHIMERA STATE}

When $\beta=0$, it is possible to calculate the chimera state exactly, at the moment of its birth from a spatially modulated drift state. Recall that all states of pure drift satisfy $\Theta(x) \equiv 0$, and equivalently, that $a$ has zero imaginary part. Hence we can seek solutions of the algebraic self-consistency equations with real values of $a$ and $c$, for $\beta=0$. At the onset of the chimera, the first locked oscillators are born. As suggested by Fig. 6, this occurs when the graph of $R(x)$ intersects the horizontal line $R=\Delta$ tangentially.

Therefore the bifurcation condition is $\Delta=R_{\max }=c+a$. Plugging this into (15) and (16), and using $\Theta(x) \equiv 0$ and $\beta=0$, we find that the self-consistency equations become

$$
c=\left\langle\frac{c+a-\sqrt{(c+a)^{2}-(c+a \cos x)^{2}}}{c+a \cos x}\right\rangle
$$

and

$$
a=A\left\langle\frac{c+a-\sqrt{(c+a)^{2}-(c+a \cos x)^{2}}}{c+a \cos x} \cos x\right\rangle .
$$

Note that both of these expressions can be rewritten solely in terms of the ratio $a / c$, which suggests a neat way to solve them parametrically.

Set $s=a / c$ and substitute into (B1) above, which becomes

$$
\begin{aligned}
c & =\left\langle\frac{1+s-\sqrt{(1+s)^{2}-(1+s \cos x)^{2}}}{1+s \cos x}\right\rangle \\
& =f_{1}(s) .
\end{aligned}
$$

So we can also write $a=s c=s f_{1}(s)$.

Similarly, the $a$ equation (16) becomes:

$$
\begin{aligned}
a & =A\langle h \cos x\rangle \\
& =A\left\langle\frac{1+s-\sqrt{(1+s)^{2}-(1+s \cos x)^{2}}}{1+s \cos x} \cos x\right\rangle \\
& =A f_{2}(s) .
\end{aligned}
$$

All other quantities of interest can also be expressed in terms of $s$. For instance, we can now substitute $a=s c=s f_{1}(s)$ into (B4) and solve for $A(s)=a / f_{2}(s)=s f_{1}(s) / f_{2}(s)$. Likewise, $\Delta=c+a=(1+s) f_{1}(s)$. In summary, the incipient chimera state can be written exactly in parametric form, as follows:

$$
c=f_{1}(s)
$$




$$
\begin{aligned}
a & =s f_{1}(s) \\
A & =s \frac{f_{1}(s)}{f_{2}(s)} \\
\Delta & =(1+s) f_{1}(s) .
\end{aligned}
$$

Since $A$ is a control parameter of the original equations (the only free one after $\beta$ has been chosen to be zero), it is desirable to reparametrize this solution in term of $A$. To do that, we invert $A(s)$ in (B5) to obtain the following series expansion for $s(A)$,

$$
s \sim \frac{16}{9 \pi^{2}} A^{2}-\frac{16}{27}\left(\frac{3 \pi^{2}-32}{27 \pi^{4}}\right) A^{3}+\mathcal{O}\left(A^{4}\right),
$$

and use that to rewrite the newborn chimera in terms of $A$ :

$$
\begin{aligned}
c & \sim 1-\frac{16}{3 \pi^{2}} A+\frac{8}{9}\left(\frac{5 \pi^{2}-32}{\pi^{4}}\right) A^{2}+\mathcal{O}\left(A^{3}\right), \\
a & \sim \frac{16}{9 \pi^{2}} A^{2}-\frac{16}{27}\left(\frac{3 \pi^{2}-16}{\pi^{4}}\right) A^{3}+\mathcal{O}\left(A^{4}\right), \\
\Delta & \sim 1-\frac{16}{3 \pi^{2}} A+\frac{8}{9}\left(\frac{7 \pi^{2}-32}{\pi^{4}}\right) A^{2}+\mathcal{O}\left(A^{3}\right) .
\end{aligned}
$$

Notice that this has exactly the form of the ansatz we postulated in (24), based on numerical experiments. As expected, it satisfies $\Delta_{1}=c_{1}$ as in (25) and gives $\Delta_{2}-c_{2}=16 /\left(9 \pi^{2}\right)$. 
[1] D. M. Abrams and S. H. Strogatz. Chimera states for coupled oscillators. Phys. Rev. Lett., 93(17):174102, OCT 2004.

[2] D. Battogtokh and Y. Kuramoto. Turbulence of nonlocally coupled oscillators in the BenjaminFeir stable regime. Phys. Rev. E, 61(3):3227-3229, MAR 2000.

[3] R. BenYishai, D. Hansel, and H. Sompolinsky. Traveling waves and the processing of weakly tuned inputs in a cortical network module. J. Computational Neuroscience, 4(1):57-77, 1997.

[4] M. C. Cross and P. C. Hohenberg. Pattern formation out of equilibrium. Rev. Mod. Phys., 65:851-1112, 1993.

[5] A. Dhooge, W. Govaerts, and Y. A. Kuznetsov. Matcont: A Matlab package for numerical bifurcation analysis of odes. ACM Trans. Math. Soft., 29(2):141-164, JUN 2003.

[6] B. Ermentrout, J. Campbell, and G. Oster. A model for shell patterns based on neural activity. Veliger, 28(4):369-388, APR 1986.

[7] B. S. Gutkin, C. R. Laing, C. L. Colby, C. C. Chow, and G. B. Ermentrout. Turning on and off with excitation: The role of spike-timing asynchrony and synchrony in sustained neural activity. J. Computational Neuroscience, 11(2):121-134, 2001.

[8] Y. Kuramoto. Chemical Oscillations, Waves, and Turbulence. Springer, Berlin, 1984.

[9] Y. Kuramoto. Scaling behavior of turbulent oscillators with nonlocal interaction. Prog. Theor. Phys., 94(3):321-330, SEP 1995.

[10] Y. Kuramoto. In S. J. Hogan, A. R. Champneys, B. Krauskopf, M. di Bernardo, R. E. Wilson, H. M. Osinga, and M. E. Homer, editors, Nonlinear Dynamics and Chaos: Where Do We Go From Here?, page 209. Institute of Physics, Bristol, UK, 2003.

[11] Y. Kuramoto and D. Battogtokh. Coexistence of coherence and incoherence in nonlocally coupled phase oscillators. Nonlin. Phenom. Complex Syst., 5(4):380-385, 2002.

[12] Y. Kuramoto and H. Nakao. Origin of power-law spatial correlations in distributed oscillators and maps with nonlocal coupling. Phys. Rev. Lett., 76(23):4352-4355, JUN 1996.

[13] C. R. Laing and C. C. Chow. Stationary bumps in networks of spiking neurons. Neural Computation, 13(7):1473-1494, 2001.

[14] R. D. Li and T. Erneux. Preferential instability in arrays of coupled lasers. Phys. Rev. A, 46(7):4252-4260, OCT 1992. 
[15] J. Medlock and M. Kot. Spreading disease: integro-differential equations old and new. Mathematical Biosciences, 184(2):201-222, AUG 2003.

[16] J. D. Murray. Mathematical Biology. Springer, Berlin, 1989.

[17] J. R. Phillips, H. S. J. van der Zant, J. White, and T. P. Orlando. Influence of induced magnetic-fields on the static properties of Josephson-junction arrays. Phys. Rev. B, 47(9):5219-5229, MAR 1993.

[18] S. I. Shima and Y. Kuramoto. Rotating spiral waves with phase-randomized core in nonlocally coupled oscillators. Phys. Rev. E, 69(3):036213, MAR 2004.

[19] S. H. Strogatz. From Kuramoto to Crawford: exploring the onset of synchronization in populations of coupled oscillators. Physica D, 143(1-4):1-20, SEP 2000.

[20] J. W. Swift, S. H. Strogatz, and K. Wiesenfeld. Averaging of globally coupled oscillators. Physica D, 55(3-4):239-250, MAR 1992.

[21] N. V. Swindale. A model for the formation of ocular dominance stripes. Proc. Roy. Soc. London B, 208(1171):243-264, 1980.

[22] D. Tanaka and Y. Kuramoto. Complex Ginzburg-Landau equation with nonlocal coupling. Phys. Rev. E, 68(2):026219, AUG 2003.

[23] S. Watanabe and S. H. Strogatz. Integrability of a globally coupled oscillator array. Physical Review Letters, 70(16):2391-2394, 1993.

[24] S. Watanabe and S. H. Strogatz. Constants of motion for superconducting Josephson arrays. Physica D, 74(3-4):197-253, 1994.

[25] K. Wiesenfeld, P. Colet, and S. H. Strogatz. Frequency locking in Josephson arrays: Connection with the Kuramoto model. Phys. Rev. E, 57(2):1563-1569, FEB 1998.

[26] A. T. Winfree. Biological rhythms and behavior of populations of coupled oscillators. $J$. Theor. Biol., 16(1):15, 1967.

[27] A. T. Winfree. The Geometry of Biological Time. Springer, New York, 1980.

[28] H. G. Winful and S. S. Wang. Stability of phase locking in coupled semiconductor-laser arrays. Appl. Phys. Lett., 53(20):1894-1896, NOV 1988. 